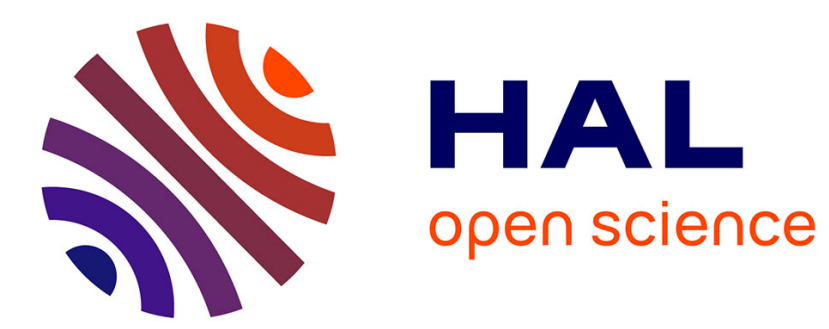

\title{
Les baleines d'Albert le Grand
}

Laurence Moulinier

\section{To cite this version:}

Laurence Moulinier. Les baleines d'Albert le Grand. Médiévales, 1992, 22-23, Pour l'image, printemps 1992, pp.117-128. halshs-00609461

\section{HAL Id: halshs-00609461 https://shs.hal.science/halshs-00609461}

Submitted on 29 May 2014

HAL is a multi-disciplinary open access archive for the deposit and dissemination of scientific research documents, whether they are published or not. The documents may come from teaching and research institutions in France or abroad, or from public or private research centers.
L'archive ouverte pluridisciplinaire HAL, est destinée au dépôt et à la diffusion de documents scientifiques de niveau recherche, publiés ou non, émanant des établissements d'enseignement et de recherche français ou étrangers, des laboratoires publics ou privés. 


\section{Les baleines d'Albert le Grand}

In: Médiévales, №22-23, 1992. pp. 117-128.

Citer ce document / Cite this document :

Moulinier Laurence. Les baleines d'Albert le Grand. In: Médiévales, N²2-23, 1992. pp. 117-128.

doi : $10.3406 /$ medi.1992.1243

http://www.persee.fr/web/revues/home/prescript/article/medi_0751-2708_1992_num_11_22_1243 
Médiévales 22-23, printemps 1992, pp. 117-128

\section{Laurence MOULINIER}

\section{LES BALEINES D'ALBERT LE GRAND}

Le fameux théologien allemand Albert le Grand, mort à plus de quatre-vingts ans en 1280 , a laissé une œuvre immense qui lui valut certes le surnom de "docteur universel » mais dont la postérité fut inégale. S'il fut parmi les premiers, selon Jacques Le Goff', à vouloir « refaire Aristote à l'usage des Latins » et s'il eut à cœur, notamment en science, de compléter par sa propre expérience ses fiches tirées des auteurs de l'Antiquité, sa réputation de zoologue n'atteignit pas celle de maître en science occulte à qui l'on attribua longtemps la paternité du Grand Albert et autres Secrets ${ }^{2}$. Pour Robert Delort en effet, une cuvre aussi importante pour l'histoire de la zoologie que son De animalibus, achevé vers 1270, ne devait exercer aucune influence durable sur les zoologues contemporains ou postérieurs et encore moins sur le public ${ }^{3}$.

Pourtant, au Moyen Age, de larges extraits de ces Animaux figurent dans de nombreux manuscrits médicaux latins et, au XVI siècle, ils attirent aussi bien l'attention du grand naturaliste suisse Conrad Gesner que celle du Suédois Olaus Magnus (Olof Mansson) qui y puise la matière de passages entiers de son Historia de gentibus septentrionalibus. Preuve, s'il en était besoin, de la vigueur d'une œuvre dont les dix-neuf premiers livres empruntent sans doute largement à Aristote $^{4}$ mais dont les sept derniers sont riches en informations tirées d'observations personnelles ou d'enquêtes menées loin des livres, pour ainsi dire sur le terrain. Albert a ainsi interrogé des chasseurs de baleines, encore très répandues en mer du Nord au XIII' siècle et p. 424.

1. Jacques Le Goff, La civilisation de l'Occident médiéval, Paris, Arthaud, 1984,

2. La paternité des Secreta mulierum lui est définitivement refusée ; mise au point sur la question par B. KUSCHE, "Zur "Secreta mulierum" Forschung ", Janus, n 62, 1975, pp. 103-123.

3. Robert Delort, Les animaux ont une histoire, Paris, Le Seuil, 1984, p. 46.

4. Danielle JACQuART et Claude ThOMASSET parlent de « vaste paraphrase de l'ensemble des œuvres zoologiques d'Aristote " (Sexualité et savoir médical au Moyen Age, Paris, P.U.F., 1985, p. 93). 
sans doute aussi près des rivages français avant que « harcelées avec acharnement », elles ne se retirent " vers des latitudes plus septentrionales " si l'on en croit Lacépède (1756-1825), autre grand lecteur d'Albert ${ }^{5}$.

L'histoire de la chasse à la baleine est en effet celle d'un déplacement progressif des hommes et des bêtes vers le nord. Pendant longtemps, on s'était contenté de traquer les baleines en les rabattant sur le rivage et les Basques furent les premiers, au Haut Moyen Age, à oser s'y attaquer en pleine mer; aussi la chasse à la baleine devintelle très tôt - dès le $\mathrm{IX}^{\mathrm{e}}$ siècle selon Yves Cohat ${ }^{6}$ - une activité primordiale pour ces pionniers. D'autres peuples les imitèrent ; mais ils devaient rester encore longtemps les maîtres incontestés du harpon et étendre leur champ d'action à mesure que les baleines se raréfiaient en baie de Biscaye pour gagner le Nord. Avec elles se déplaçaient les lieux de chasse, en un mouvement qui culmina au XVI ${ }^{\text {e }}$ siècle lorsque la découverte des côtes du Spitzberg, véritable " mine " où les Hollandais mirent rapidement sur pied la toute première industrie baleinière, sonna le glas de la suprématie basque.

Au XIII siècle en tout cas, du vivant de notre auteur, les baleines sont assez nombreuses en mer du Nord pour faire l'objet d'une chasse dont il nous décrit les techniques, l'organisation et les enjeux. À cet égard, le portrait qu'il brosse du " cète " au premier chapitre du livre XXIV de ses Animaux nous a paru assez précieux pour l'histoire des hommes et de la zoologie pour en proposer une traduction, qui suit le texte de l'édition établie par Hermann Stadler?

Ce texte posera sans doute aux cétologues des questions auxquelles nous ne sommes pas en mesure de répondre : est-ce le cachalot ou la baleine franche dont Albert évoque la tête si recherchée pour sa graisse ? Comment notre auteur, qui prend soin de signaler comme telles ses informations livresques et d'éliminer les plus invraisemblables au profit des données de l'expérience, peut-il évoquer les yeux gigantesques ou les longs cils de la baleine ? Et quel animal se cache derrière ce " cète " dont la bouche est "faite pour sucer ", " non pour mâcher " ? Apparemment pas un cétacé, puisque sa bouche semble également dépourvue des dents caractéristiques du sous-ordre des Odontocètes et des fanons spécifiques des Mysticètes...

Un tel texte nous rappelle entre autres que les connaissances scientifiques dont nous disposons actuellement en la matière sont récentes, et qu'elles furent précédées par un savoir issu de l'expérience des marins qui, pendant longtemps, ont été les seuls à avoir effectivement

5. LACÉPÉDE, Histoire naturelle des cétacées [sic], Paris, Plassan imprimeur, l'an XII de la République, p. 73. p. 48

6. Yves Cohat, Vie et mort des baleines, Paris, Découvertes Gallimard, 1986,

7. Albertus Magnus de Animalibus libri $X X V I$, nach der Cölner Urschrift herausgegeben von Hermann Stadler, Münster, 1916, 2. Band, pp. 1522-1525. 
pu observer des baleines vivantes. Aussi leurs récits sur les mœurs des différentes espèces, le lieu et le moment propices à l'attaque ou la meilleure façon de lancer le harpon devaient-ils rester la base d'une science des cétacés jusqu'au XVIII siècle, époque où Linné, Cuvier, Daubenton ou Lacépède l'enrichirent d'un nouveau savoir, un savoir né "loin des océans" pour reprendre un mot d'Yves Cohat ${ }^{8}$.

Il serait donc vain de chercher dans ce texte la préfiguration de certaines de nos catégories actuelles : les notions de " mammifère " ou de " cétacé " sont étrangères à Albert qui, à la suite de Pline, considère le " cète " comme un poisson", et établit un lien entre cachalot et morse ou dauphin et requin mais pas entre baleine et dauphin.

Nous avons cependant tenu à faire suivre notre traduction par celle du chapitre consacré à un autre cétacé - le dauphin - dans le même livre, quelques pages plus loin ${ }^{10}$. Un tel rapprochement fait certes preuve d'un sens de la «famille » qui est le nôtre et pas celui de l'auteur ; mais il est dû avant tout au désir de mettre en lumière à la fois l'unité d'un thème - la pêche comme mise en contact de l'homme et des animaux marins - et la complexité d'un auteur. Albert le Grand a en effet une approche plurielle du monde animal et, s'il privilégie l'expérience vécue dans le cas du " cète ", il fait aux récits des Anciens à propos du dauphin une place qui n'exclut ni le doute prudent ni, parfois, une grande crédulité. Aussi ces deux textes ont-ils à nos yeux un double intérêt : outre la vie et la mort des baleines au XIII ${ }^{e}$ siècle, c'est aussi la naissance d'une véritable Naturforschung, avec ses doutes et ses impasses, que l'on peut y lire".

\section{La baleine}

« Le cète ${ }^{12}$ est le plus grand poisson qu'on ait jamais vu, et sa femelle est appelée baleine ${ }^{13}$.

8. Yves Cohat, op. cit., p. 29.

9. Il en fait certes le numéro un de cette catégorie, comme la baleine Jasconius de la Navigation de saint Brandan, récit de voyage merveilleux du Haut Moyen Age ; cf. J. Le Goff, "Le merveilleux nordique médiéval ", in Pour Jean Malaurie, 102 témoignages en hommage d 40 ans d'études arctiques, Paris, Plon, 1990, pp. 21-28.

10. Albertus Magnus de Animalibus libri XXVI, op. cit., pp. 1530-1531.

11. Sur cet aspect de l'œuvre d'Albert le Grand, citons, entre autres, Heinrich Balss, Albert der Grosse als Zoologe, München, 1928, qui a analysé l'attitude d'Albert envers ses sources: ainsi Pline, cité 65 fois, est critiqué 11 fois, et Solin, mentionné 22 fois, est critiquẽ 5 fois. Cf. aussi Paul Hossfeld, Albertus Magnus als Naturphilosopher und Naturwissenschaftler, Bonn, 1983, et J.A. WeISHEIPL ed., Albertus Magnus and the sciences, Commemorative Essays, Toronto, 1980.

12. Chez Pline (Hist. nat. 32, 10), ce mot, sous sa forme cetos, ne désigne pas une espèce particulière mais une bête marine de grande taille, baleine, dauphin ou même thon. Nous le rendons ici par cète, puisque c'est sous cette forme qu'il apparaît, à l'époque d'Albert, dans le Livre du Trésor que le Florentin Brunetto LATINI, mort en 1294, écrivit directement en français.

13. B. LATINI en fait un synonyme de " cète ": "Cete est un grans poisson que li plusor apelent balaine " (Petit bestiaire, éd. G.L.M., 1967, p. 13 tandis que Guil- 
Il y a de nombreuses variétés de cètes : les plus grands ont des poils $^{14}$, ceux qui ont la peau lisse sont plus petits et, dans notre mer, on peut voir et pêcher deux sortes de cètes à peau lisse. Le premier, quand il ouvre la bouche, découvre de très grandes dents, longues en général de deux coudées, parfois de trois et même de quatre, mais le plus souvent d'une seule coudée; deux de ces dents en particulier - les dents canines - sont plus longues que les autres, et creuses au-dessous comme une corne. Elles ressemblent aux dents de l'éléphant et à celles du sanglier qu'on appelle broches ${ }^{15}$, et visiblement elles servent à se battre. Ce genre de cète $\mathrm{a}$, pour sa part, une bouche qui lui permet de mâcher.

L'autre en revanche - qu'on a vu encore récemment - a, comme la murène, une bouche sans dents faite pour sucer ; il est un peu plus petit, et a une chair bien meilleure que le précédent.

Ni l'un ni l'autre ne possède de branchies, c'est-à-dire qu'ils respirent comme les dauphins, grâce à un tuyau ${ }^{16}$.

En revanche ces deux sortes de cètes à peau lisse ont une peau noire et épaisse et, au-dessus des yeux, qui sont très grands (l'orbite d'un seul œil peut contenir quinze hommes, parfois même vingt) ${ }^{17}$, des formations cornées semblables à des cils de huit pieds de long, un peu plus ou un peu moins selon la taille du poisson; et ces formations cornées, au nombre de 250 au-dessus de chaque œil, ont l'aspect d'une de ces grandes faux avec lesquelles on coupe le blé ${ }^{18}$.

laume le Clerc, auteur d'un Bestiaire divin vers 1210 , distingue « l'esturgeon, la baleine, le turbot, le cachalot, le marsouin » et " un monstre malfaisant qui se nomme Cetus en latin. " (Bestiaires du Moyen Age, prés. et trad. G. Bianciotto, Paris, Stock Plus, 1980 , p. 105).

14. Conrad Gesner, à la suite d'Olaus Magnus, cite le Bartwal (cetus barbatus) et le Haarwal (cetus capillatus), illustrations à l'appui (cf. Icones animalium marinorum, ed. à la suite des Icones avium, editio secunda, Tiguri excudebat C. Froschoverus, MDLX, pp. 180-181). Plus loin, dans ses "Addenda " (p. 368), il précise : "qui Morsi seu Rosmari vocantur (...) caput habent bovis instar et pellem hirsutam (quare ab Alberto Magno hirsuti vocantur) cuius pili, culmi frumentacei crassitudine, late diffluunt ".

15. Culmi dans notre texte.

16. C'est l'évent, déjà connu d'Aristote qui évoque "le dauphin et la baleine ainsi que les autres cétacés qui ont un tuyau ou évent, au lieu de branchies " et précise : "Le dauphin a le tuyau sur le dos, tandis que la baleine l'a sur le front " (Histoire des animaux, trad. J. Barthélémy-Saint-Hilaire, Paris, 1883, livre VI, chapitre XI, p. 304 et I, IV, p. 25).

17. Affirmation surprenante : les yeux des cétacés sont franchement petits et leur diamètre n'est souvent " que la $192^{\bullet}$ partie de la longueur totale du cétacée (sic) " selon Lacépède, op. cit., p. 17, ce qui n'empêche pas Gesner de 'reprendre ce qu'en dit ici Albert (Icones animalium marinorum, op. cit., p. 180).

18. Les yeux de la baleine sont dénués de cils et la description d'Albert évoque bien plus... les fanons, lames cornées frangées sur leur bord inférieur, spécifiques du sous-ordre des Mysticètes à qui ils servent de piège à plancton.

Cf. ce que dit Lacépède des fanons, " réunis et comme collés par une substance gélatineuse qui, lorsqu'elle est sèche, lui donne presque toutes les propriétés de la corne (c'est nous qui soulignons), dont il (le fanon) a l'apparence. Chacun de ces fanons 
Leur base, enracinée dans la peau, est plus large que la pointe par laquelle elles se terminent et sont séparées les unes des autres; et elles ne se dressent pasi à la verticale par rapport au corps mais reposent, couchées, entre la racine de l'œil et la tempe du poisson, de sorte qu'elles semblent ne former qu'un seul os, large comme un grand van ; notre poisson s'en sert pour protéger ses yeux en cas de gros temps.

Sa bouche est immense et quand il respire, il recrache une quantité d'eau assez grande pour remplir des canots et les couler ${ }^{19}$. Il a de grandes nageoires, comme le dauphin, et une queue formant une fourche large de plus de $\mathbf{2 4}$ pieds quand le poisson a atteint sa taille adulte. Quant aux côtes, elles sont aussi longues et recourbées que le poisson est gros, et leur masse évoque les madriers des grandes maisons - j'entends par madriers ces poutres qui soutiennent la charpente sur laquelle on fixe les tuiles du toit.

Les Anciens racontent que ce poisson occupe, par la largeur de son ventre, quatre arpents de terre ${ }^{20}$, mais aucun des mariniers qui ont vu des cètes en abondance n'a jamais pu nous le confirmer. Quoi qu'il en soit, ce que nous pouvons garantir n'est pas moins impressionnant : une fois dépecé, un de ces poissons peut remplir 300 chariots entre sa chair et ses os ; il est certes rare qu'on en prenne d'aussi grands, mais ceux que l'on pêche ici remplissent souvent entre cent cinquante et deux cents chariots.

Comme le dauphin, ce poisson a sa verge et ses testicules à l'intérieur du corps, et il fait sortir sa verge lors de l'accouplement; la femelle a pour sa part une vulve très semblable à celle de la femme ${ }^{21}$. Quand

est d'ailleurs très aplati, allongé, et très semblable par sa forme générale à la lame d'une faux " (op. cit., p. 11).

Gesner rapproche de cette description celle que fait Olaus Magnus des " cornes " du Bartwal : "XIV cornibus radiatum caput apparet, cornua utrinque ab oculo incipiunt, et per oculum transeunt : nescio quam recte. Albertus enim cetis nostrorum marium appendices quasdam esse scribit (circa oculos) ciliorum instar, corneas, octo fere pedes longas " (Icones animalium marinorum, op. cit., p. 180). Dans ses " Addenda " (p. 370) l'existence de ces " appendices" est ainsi justifiée : "Haec simul cohaerent ad oculorum protectionem tempore tempestuoso, aut cum alia eum invaserit bellua inimica. Neque mirum quod tot cornua, licet satis molesta, habeat, cum inter oculos in fronte spatium sit $X V$ vel $X X$ aut amplius pedum ".

19. Cf. Solin, 52, 42 : « Physeteras nuncupantur qui (...) super antennas se navium extollunt haustosque fistulis fluctus ita eructant ut nimbosa alluvie plerumque deprimant alveos navigantium " (Collectanea rerum memorabilium, rec. Th. Mommsen, Berlin, 1864, p. 211). Lacépède ne dit pas autre chose : « La baleine fait sortir par ces évents un assez grand volume d'eau pour qu'un canot puisse en être bientôt rempli » (op. cit., p. 8).

20. Affirmation qui se trouve chez Pline, abondamment reprise par la suite. Cf. Solin, 52, 42 : "Indica maria balaenas habent ultra spatia quattuor jugerum " (op. cit., p. 211) et GESNER : « Magnitudinem balaenarum Plinius quatuor jugerum, id est nongentorum sexaginta pedum esse meminit " (Historiae animalium liber IIII qui est de Piscium et Aquatilium animantium natura, Tiguri apud C. Froschoverum, MDLVII, p. 231).

21. Les cétacés ont une forme hydrodynamique accomplie, grâce notamment à l'effacement d'aspérités habituelles sur le corps des mammifères : chez le mâle, les tes- 
ils s'accouplent, la femelle se place sous le mâle, comme le font les humains et les dauphins : mais leur coït est bref, comme chez tous les animaux dont les testicules sont à l'intérieur ${ }^{22}$. Ce qui reste alors du sperme - car il est émis en très grande quantité, et la vulve de la baleine ne peut tout contenir - est très recherché par les médecins : c'est ce qu'on appelle l'ambre ${ }^{23}$, souverain contre la goutte et la paralysie. Au demeurant, si verge et testicules sont à l'intérieur, c'est pour que ces organes ne gênent pas le cète quand il nage et pour que le contact permanent avec l'eau froide ne soit pas fatal à leur vigueur.

D'aucuns prétendent qu'après un seul coït, le cète ne peut plus s'accoupler avec la baleine, devient impuissant et gagne alors les profondeurs de la mer où il engraisse tellement qu'il devient aussi gros qu'une île ; mais je doute que cela soit vrai, et les plus expérimentés ne racontent rien de tel ; en revanche, quand des cètes se battent pour leurs femelles et leurs petits, il est vrai que l'animal vaincu gagne le fond des eaux, où la peur le fait rester un certain temps; et comme il est immobile, il engraisse beaucoup ; et ce poisson, qui a du lard dans le dos comme le porc, a beaucoup de gras dans la tête, surtout autour de la moelle cérébrale.

J'ai été contemporain de plusieurs captures ; en Frise, près d'un lieu du nom de Stauria, on prit un cète dont la tête, percée au moyen d'un pieu enfoncé dans l'œil, produisit onze jarres de graisse, si lourdes qu'un homme pouvait à peine en porter une seule. Et cette graisse et ces jarres, je les ai vues de mes propres yeux : et je peux dire qu'une fois purifiée, c'est une graisse très claire et très brillante. Un autre cète fut capturé au large de Maastricht, en Hollande, et sa tête rendit $\mathbf{4 0}$ jarres de graisse. Le lard de ce poisson est ce qu'on appelle graspois $^{24}$.

ticules sont intra abdominaux et le pénis logé dans la paroi du corps; chez la femelle, la vulve est associée à l'anus dans une poche. Cf. LACEPEDE, op. cit., p. 26 : « La vulve, qui a son clitoris, son méat urinaire et son vagin ".

22. Ȧ comparer avec ce que dit Aristote de l'accouplement des dauphins « et de tous les cétacés »: " le mâle saute sur la femelle qu'il frôle et la durée de l'acte n'est ni trop courte ni trop longue " (Histoire des animaux, op. cit., p. 131).

23. Spermaceti pris au pied de la lettre ? Cette substance huileuse, également appelée "blanc de baleine ", est improprement nommée puisqu'on la trouve dans la tête de l'animal. Cf. LACÉPédE : "Combien de raisons n'avons-nous pas pour rejeter les dénominations erronées de blanc de baleine, sperma ceti, etc., pour adopter "adipocire" proposée par Fourcroy?" (Histoire naturelle des cétacées, op. cit., p. 185). Quant à l'ambre gris, il provient des concrétions intestinales du cachalot.

24. Craspois ou graisse de baleine : l'Église en autorisera son utilisation en cuisine en carême en 1300, à la place des huiles méridionales, toujours rances lorsqu'elles arrivent dans le Nord (Cf. Jean-Noël BIRABEN, "Épidémies, hygiène et santé publique au Moyen Age ", Colloque international d'histoire de la médecine médiévale, Orléans, 1985, tome 1, p. 79). 
La baleine a un petit à la fois, qu'elle nourrit et qui suit sa mère jusqu'à l'âge de trois ou quatre ans ${ }^{25}$.

Lorsque ce poisson se trouve bloqué par les bateaux qui l'encerclent, il plonge, puis réapparaît subitement à la surface de l'eau et coule les bâtiments. On arrive cependant très souvent à le prendre quand l'appétit lui fait suivre un banc de harengs : il se retrouve alors souvent sur le rivage, et ne parvient pas à regagner l'eau. C'est ainsi qu'un cète s'est échoué récemment sur la côte frisonne : quand on le découvrit, on n'eut qu'une crainte : et s'il regagnait aussitôt les flots ? Alors, de peur de perdre le poisson, les habitants l'attachèrent avec tout ce qu'ils purent trouver comme cordages sur leur île, fichant des pieux profondément en terre et liant l'extrémité des cordes aux pierres, aux maisons voisines, et à d'autres bâtiments. Mais la marée montante vint au secours du poisson et brisa ses entraves : le cète encore empêtré regagna le large, à la grande tristesse des habitants qui pleuraient la perte de leurs cordages. Mais le cète était à jeun et deux jours plus tard, ayant suivi - avec ses cordes ! - un banc de harengs, il s'échoua au même endroit ; cette fois, les habitants récupérèrent leurs cordes et tuèrent, puis dépecèrent le poisson. Et quand on se mit à découper son cou, brisé par le poids de la tête, il craqua avec autant de fracas qu'une maison qui s'écroule.

Les mariniers qui chassent les cètes dans nos eaux les capturent de deux manières. Voici la première : les pêcheurs se rassemblent en grand nombre et montent trois par trois à bord de petits canots qui voguent vers l'endroit où l'on suppose qu'il y a des cètes; deux de ces hommes s'occupent de la navigation, le troisième se tient debout, prêt à frapper avec un instrument dont le manche est en sapin bois choisi pour sa légèreté - et au bout duquel se trouve, près de la main du harponneur, un trou dans lequel passe une corde très solide et très longue. Celle-ci repose lovée sur le fond du bateau, et peut ainsi se dérouler aisément sans risque de fausse manœuvre, et suivre l'instrument ${ }^{26}$ auquel est fixé un de ses bouts. L'autre extrémité

25. Le fœetus est généralement unique, et la mère allaite son petit ; la plupart des baleines atteignent leur maturité sexuelle entre la troisième et la cinquième année.

26. Le harpon doit être en fer, d'après les encyclopédies contemporaines : " quoddam ad instar rastri ferreis dentibus accuminatum clam in dorsum cethi proiciunt clamque diffugiunt " (Thomas DE CANTIMPRÉ, Liber de natura rerum, éd. De Gruyter, Berlin/New York, 1973, p. 233 et Vincent de BeauvaIs, Speculum naturale, XVIII, 42, Douai 1624, col. 1275). Cf. aussi le témoignage d'un anonyme anglais, vers 1200 , édité d'après un manuscrit de la Bodleian Library par B. LAwN, The Prose Salernitan Questions, Londres, 1979. Je remercie Alain Boureau d'avoir attiré mon attention sur le texte de la page 109 : "Queritur quare balena non calibe sed ferro inciditur ? Solutio. Caro balene callosa est, que licet cum calibe melius deberet incidi, cum calebs acute sit substantie deberet inferre mortem. Sed dum calibe inciditur, se contrahit et humores in magna quantitate concurrunt ad vulnus, et extenditur locus ille, unde ex incisione non potest ****** telum penetrare. Sed vulnus ferro factum leve fit respectu illius, unde non tanta sit spirituum immutatio, quare non tanta humorum attractio. Preterea calebs frigidior est ferro. Cum balena superficiem habeat valde duram, frangitur calebs. Ferrum dulcius est, 
de cet instrument, quant à elle, est formée d'un triangle semblable à une flèche barbelée, dont la pointe est très acérée et parfaitement lisse, pour une meilleure pénétration ; les deux bords qui se rejoignent à la pointe sont tranchants comme un rasoir très aiguisé ; le triangle lui même est mince et sa surface très lisse ; enfin, du milieu du côté opposé à la pointe part un morceau de fer qui lui est perpendiculaire, long d'une coudée ou un peu plus ; il se termine par un trou, dans lequel entre le manche que nous évoquions ${ }^{27}$.

Dans chaque barque, un des trois mariniers se tient donc debout, et brandit cet instrument ; dès que le cète est en vue, ils hèlent les autres équipes, qui sont elles aussi prêtes pour l'action, et leur demandent du renfort.

Le cète, en agitant l'eau, a chassé les poissons du fond vers la surface, où il nage ; c'est alors que les mariniers le frappent, le plus profondément possible, pour reculer aussitôt en laissant leurs harpons fichés dans les blessures ${ }^{28}$. Si le cète prend le large dès qu'il a été frappé, les pêcheurs coupent les cordages et n'ont plus qu'à regretter la peine et l'argent perdus. Si en revanche l'animal plonge immédiatement, c'est qu'il est sérieusement blessé et va se frotter contre le fond à cause du sel qui pénètre dans ses plaies ; ce faisant, il enfonce de plus en plus les pointes dans sa chair, et le sang qui jaillit sans attendre en bouillonnant à la surface est le signe des souffrances du poisson qui, affaibli par la perte de son sang, cherche un terrain solide et s'approche peu à peu du rivage.

Tous les habitants, montés dans des barques et munis de piques, attendent qu'il apparaisse ; et, dès qu'il se montre, il est encerclé et mis à mort.

L'autre méthode employée pour la capture des cètes est quasiment identique, à cela près que le harpon est lancé non pas à la main, mais au moyen d'une baliste très puissante dans laquelle on fait passer la corde dont nous avons déjà parlé 29 .

ideo non frangitur sed penetrat. Vel aliter; cuiuscunque complexionis est illud animal, superficies tamen sicca est. Cum ergo calibe inciditur, siccitas calibis augmentatur unde cito frangitur. Ferrum vero aliquantulum habet humiditatis in respectu calibis. Humiditate sua duritiem relaxat et acumine suo usque ad interiora penetrat, unde solutionem continuitatis infert et sic mortem. "

27. Cf. LACÉPÉDE, op. cit., p. 83 : " Ce fer, ou le dard proprement dit, se termine par une douille de près d'un mètre de longueur, dans laquelle on fait entrer un manche très gros long de deux ou trois mètres. On attache au dard même ou à sa douille la ligne, qui est faite du plus beau chanvre. "

28. Cf. LACÉPÉDE, op. cit., p. 87 : "Dans les premiers temps de la pêche à la baleine, on approchait le plus possible de cet animal avant de lui donner un coup de harpon. Quelquefois même le harponneur ne l'attaquait que lorsque la chaloupe était arrivée sur le dos du cétacé. "

29. Cf. encore LACÉPÉDE, op. cit., p. 88 : " Albert rapporte que de son temps des pêcheurs au lieu de jeter le harpon avec la main, le lançaient au moyen d'une baliste. " 
Quant aux cètes qui ont des poils, ils ont des broches ${ }^{30}$ très longues grâce auxquelles ils se suspendent aux rochers quand ils dorment ; c'est alors que le pêcheur peut s'approcher et qu'il sépare, autant qu'il est possible, la peau et le lard près de la queue du poisson ${ }^{31}$; puis, dans le trou ainsi ménagé entre peau et lard, il fait passer une corde très solide qu'il attache ensuite à des anneaux fixés dans la roche, à des pieux très robustes ou à des arbres. Enfin, au moyen d'une grande fronde, il lance de grosses pierres en visant la tête du poisson, pour le tirer de son sommeil ; ainsi réveillé, l'animal veut s'enfuir et le premier geste qu'il esquisse lui arrache la peau du dos, depuis la queue jusqu'à la tête. On capture ensuite le cète non loin de l'endroit où il a laissé sa dépouille, nageant à bout de forces ou gisant à demi mort sur le rivage ${ }^{32}$.

On fait avec cette peau des courroies très solides pour les poulies qui soulèvent de très lourdes charges, et il $y$ en a toujours en vente au marché de Cologne ${ }^{33}$.

Voilà ce que nous connaissons par expérience de la nature des cètes; nous n'avons pas tenu compte des récits des Anciens qui ne correspondent pas à la réalité. "

\section{Le dauphin}

«On trouve dans la mer de nombreuses sortes de dauphins; ceux que l'on y voit le plus souvent ont la peau noire, une tête courte et, dans la bouche, des dents alignées comme le sont les molaires du porc. Cet animal aime l'homme et sa propre espèce : ainsi, quand les jeunes se promènent en groupe, deux dauphins adultes les encadrent, et

30. Cf. note 15 .

31. Sans doute le morse. Cf. noté 3 et ce qu'en dit Olaus Magnus, cité par GesNER (Icones animalium marinorum, op. cit, , p. 178) : "Rosmarus (inquit) est bellua marina, ad magnitudinem elephantis. Litorum montes ascendit et gramine pascitur. Somni gratia dentibus se a rupe suspendit et adeo profunde dormit ut piscatores laqueis et funibus victum comprehendant ".

32. Témoignage très semblable cheż GESNER (Icones animalium marinorum, op. cit., p. 179) : "Est in Orchadibus (inquit Hector Boethius, in descriptione Scotiae) ingens quidam, mole sua vel maximum equum excedens cetus, portentosa quadam somniculitate. Is in cotem aliquam aqua extantem exiliens, dentibus (quos maximos robustissimosque habet) partem aliquam cotis extantem atque asperam comprehendit, ac mox in gravissimum solvitur soporem. Tum nautae, si qui forte praetervecti pendentem eminus cernunt, appellentes, anchoram jaciunt, valentissimo ad eum rudente ligato, ac scapha ad belluam adnavigant, circa caudam ejus cutem atque carnem aliquantum excavantes, quo firmius adligatus rudens mox salientem retinet, quo facto strepitum clamoremque continuo nautae omnes extollunt, lapidibus in eum plurimis coniectis."

33. Cf. GeSNER, Iconés animalium marinorum, op. cit., p. 179 : "Cute ad retinacula utuntur quippe cum fortissima sit, ac rumpi perdifficilis, nec longissimi attritu temporis deteratur ». D'après lui, le morse est également chassé pour ses défenses : « Expetitur autem maxime propter dentes, qui preciosi sunt apud Scythas, Moschos scilicet, Ruthenos ac Tartaros " (ibidem, p. 368). 
si un dauphin vient à mourir, les autres protègent son corps jusqu'à ce que la mer elle-même l'ait rejeté, afin qu'il ne soit pas mangé par d'autres poissons.

En Méditerranée - cette grande mer qui baigne les deux côtés de l'Italie -, quand les pêcheurs prennent la mer, un troupeau de dauphins se forme pour les accompagner. Arrivés au large, ils se disposent en couronne autour des poissons, qu'ils poussent vers les filets en resserrant leur cercle. Les Italiens, en effet, ne pêchent pas plus le dauphin qu'ils n'en mangent : bien au contraire, ils donnent aux dauphins une partie des poissons qu'ils ont pêchés ! Et les Italiens appellent ces dauphins tumberellos ${ }^{34}$ parce qu'ils sautent devant les navires en crachant de l'eau ${ }^{35}$.

Quand cet animal se réfugie à la surface de l'eau, c'est signe que la tempête est proche ${ }^{36}$.

On dit qu'il vit très vieux, on raconte même qu'un dauphin qu'on avait amputé de sa queue vécut plus de cent ans ${ }^{37}$. Il s'accouple tous les dix mois ${ }^{38}$ et son coït est bref car ses testicules et sa verge sont à l'intérieur de son $\operatorname{corps}^{39}$ : la femelle, couchée sous le mâle, reçoit la semence dans sa vulve, semblable à celle de la baleine et de la femme.

34. LACÉPÉDE note que le dauphin est appelé : " bec d'oie, simon, camus, delfino en Italie, tumberello par les Italiens, Delphin en Allemagne " (Histoire naturelle des cétacées, op. cit., p. 250).

35. "Tumbantes ante naves ", dans notre texte. Du Cange, dans son Glossarium, mentionne un verbe " tombare : saltare, exsilire more histrionis, nostris alias thumer, tumer, tumber" (p. 603). Solin exprime la même idée : "Ante omnia nihil velocius habent maria, sic ut plerumque salientes transvolant vela navium " (C. JULII SolINI Polyhistor, vel rerum toto orbe memorabilium thesaurus, Paris, 1621, p. 148), à la suite de Pline, Hist. nat., 9, 20. Gesner cite le mot "tumberellos ", mais en l'écorchant : "Nonnulli Delphinos, Berellos vocant : eo quod (ut Alberti verbis utar) ante naves aquam evomunt " (Icones animalium marinorum, op. cit., p. 163).

36. Cf. Pline, Historia naturalis, XVIII, 87 : « Praesagiunt et animalia : delphini tranquillo mari lascivientes flatum ex qua venient parte, item spargentes aquam ; idem turbato tranquillitatem. " Voir aussi Brunetto LATINI : "Et par eulx aperçoivent li marinier la tempeste qui doit venir, quand ils voient le dalfin fuir parmi la mer, et trebuchier soi en fuiant, come se la foudre le chaçast " (Petit bestiaire, op. cit., p. 16).

37. Même expérience chez ARISTOTE VI, XI, (op. cit., p. 306) mais pour une durée moindre : "On en a vu vivre jusqu'à 25 ans et même 30 . Les pêcheurs coupent la queue de quelques-uns et les laissent aller, assurés de connaître par là quel âge ils peuvent avoir. " On la retrouve chez SOLIN (Collectanea rerum memorabilium, op. cit., p. 88) : "In tricesimum annum vivunt, quod exploratum est in experimentum caudis amputatis ", que Brunetto Latini se contente de traduire (Petit bestiaire, op. cit., p. 17).

38. Même durée chez SolıN, 12, 4, à propos de la gestation : "decimus mensis maturum facit partum ", et B. LATINI, op. cit., p. 16 : «Et sachiez que li dalfins engendrent filz, non pas œufs, et les portent dix mois. " La gestation dure effectivement de dix à douze mois selon les espèces et ne semble avoir lieu que tous les deux ou trois ans.

39. Cf. ce qui est dit de la baleine supra, note 21 . 
Cet animal aime la musique ${ }^{40}$. C'est ainsi qu'un joueur de cithare du nom d'Arion qui était tombé à l'eau fut recueilli par des dauphins qui le portèrent jusqu'au rivage ${ }^{41}$. À une autre époque, alors que le roi de Carie avait pris un grand dauphin, ses congénères avaient suivi en foule jusqu'au rivage le dauphin captif, en manifestant leur douleur ; voyant cela, le roi de Carie ordonna la libération du captif, que la troupe de dauphins retrouva avec des bonds de joie et emmena loin du rivage ${ }^{42}$. On dit aussi que si un homme tombe à l'eau alors qu'il a mangé du dauphin, les dauphins, s'il y en a, le dévorent à leur tour. Mais s'il n'a rien de tel dans l'estomac, les " dauphins le ramènent au rivage, même mort.

Sous le règne d'Auguste, il y avait, dit-on, un enfant qui élevait un dauphin : l'animal mangeait dans sa main, promenait l'enfant en mer en l'emmenant où il voulait et le ramenait sur le rivage ${ }^{43}$. Lorsque l'enfant mourut, et que le dauphin ne le vit plus paraître sur le rivage comme il en avait l'habitude, le dauphin mourut à son tour, de chagrin.

Un dauphin et un enfant du nom d'Henanus ont vécu une histoire tout à fait semblable, mais cette fois en Afrique, dans la baie d'Hippo ${ }^{44}$, si l'on en croit les récits persans.

Mais, dans la mer qui baigne notre pays, on pêche le dauphin pour le manger, et c'est pourquoi il fuit l'homme.

Il y a par ailleurs un autre genre de dauphin, dont on dit qu'il a une bouche au milieu du corps ${ }^{45}$, et qu'il est si rapide qu'aucun

40. Cf. Solin, Polyhistor, op. cit., chap. XVIII, p. 149 : « Mulcentur musica, gaudent cantis tibiarum ".

41. Poète lyrique de l'Antiquité sauvé par un dauphin ; cf. Solin, Polyhistor, op. cit., p. 151 et LACÉPÉDE, op. cit., p. 283 : " On a conservé une allégorie touchante... l'aventure d'Arion qui, menacé de mort par les féroces matelots du navire sur lequel il était monté, se précipita dans la mer, fut accueilli par un dauphin que le doux son de sa lyre avait attiré et fut porté jusqu'au port voisin par cet animal attentif. "

42. Cf. LACÉPEDE, op. cit., p. 273 : " On raconte, dit Aristote, qu'un dauphin ayant été pris sur le rivage de la Carie, un grand nombre de cétacés de la même espèce s'approchèrent du port et ne regagnèrent la pleine mer que lorsqu'on eut délivré le captif qu'on leur avait ravi." "

43. Cf. SolIN, Polyhistor, op. cit.; chap. XVIII, p. 149 : "Divo Augusto principe, in Campania delphinem puer fragmentis panis primo illexit, et in tantum consuetudo valuit, ut alendum se etiam manibus ipsius crederet. (...) " Mais l'anecdote est située au bord du lac Lucrin, près de l'actuelle Pouzzoles, selon LACÉPĖdE (op. cit., p. 276) : "Mécenas Fabius et Flavius Alfius ont écrit dans leurs chroniques suivant Pline qu'un dauphin qui avait pénétré dans le lac Lucrin recevait tous les jours du pain que lui donnait un jeune enfant (...). "

44. Hippo Diarrhytus, c'est-à-dire l'actuelle Bizerte. Sur cette ville, voir Pline, 5, 23. L'histoire se retrouve chez Solin, Polyhistor, op. cit., p. 150 : «In Africano mox littore apud Hipponem Diarrhyton Delphin ab Hipponensibus pastus tractandum se praebuit, impositos quoque frequenter gestavit (...). "Cf. LACÉPĖDE, op. cit., p. 274 : "Pline a écrit qu'en Barbarie, auprès de la ville de Hippo Diarrhyte, un dauphin s'avancait sans crainte vers le rivage (...). ")

45. Cf. Solin, Collectanea rerum memorabilium, op. cit., p. 88 : "Ora non quo ceterae beluae loco habent, sed ferme in ventribus" et sa traduction par Brunetto 
poisson ne pourrait lui échapper s'il ne se retournait pas pour attraper sa proie, de sorte que sa bouche n'est plus en bas mais en haut et qu'alors les poissons peuvent s'enfuir. Cet animal déteste si fort ses petits que le mâle les dévorerait si la femelle ne les cachait pas. Elle les cache effectivement et les emmène avec elle jusqu'à ce qu'ils aient atteint leur maturité, mais elle se met ensuite à les haïr à son tour et les dévorerait ${ }^{46}$ comme le mâle s'ils n'avaient pas acquis assez de forces pour se défendre.

Certains, enfin, parlent d'une autre variété de dauphin qui habite le Nil et porte sur le dos une crête aussi tranchante qu'une $\operatorname{scie}^{47}$; et il tue les crocodiles qui nagent à la surface de l'eau et dont le ventre est mou, en les éventrant avec sa crête ${ }^{48}$. "

LATINI, op. cit., p. 17 : « Et lor bouche n'est pas là où li autre poissons les ont, mais est près du ventre, contre la nature. " On pense au requin, dont le rostre reporte la bouche en position ventrale.

46. Albert reproduirait ici des erreurs commises par Thomas de Cantimpré qui copiait lui-même de travers Solin : là où Solin écrit " èdunt ", Thomas lit "édunt " et Albert le suit et emploie, comme lui, le verbe devorare (démonstration détaillée par Pauline AIKen, "The animal history of Albertus Magnus and Thomas of Cantimpré ", Speculum, avril 1947, vol. XXII, n², pp. 205-225.

47. Cf. à nouveau Solin, Polyhistor, op. cit., p. 270 : « Est et Delphinum genus in Nilo, quorum dorsa habent cristas", repris entre autres par Isidore (Étymologies, livre XII, 6, 11) : "Est et delphinum genus in Nilo dorso serrato, qui crocodillos tenera ventrium secantes interimunt" et B. LATINI (Bestiaires du Moyen Age, op. cit., p. 178) : "Et sachez que dans le fleuve Nil, il existe une espèce de dauphins qui portent sur le dos une nageoire dorsale semblable à une scie, à l'aide de laquelle ils tuent le crocodile. "

48. Selon Cuvier, ce dauphin à aiguillon dorsal serait l'aiguillat. 